\title{
Photon Phase Shift at the Few-Photon Level and Optical Switching by a Quantum Dot in a Microcavity
}

\author{
L.M. Wells,${ }^{1,2, *}$ S. Kalliakos, ${ }^{1}$ B. Villa,,${ }^{1,2}$ D.J.P. Ellis, ${ }^{1}$ R.M. Stevenson, ${ }^{1}$ A.J. Bennett,,${ }^{1} \dagger$ \\ I. Farrer, ${ }^{2, \$}$ D.A. Ritchie, ${ }^{2}$ and A.J. Shields ${ }^{1}$ \\ ${ }^{1}$ Toshiba Research Europe Limited, Cambridge Research Laboratory, 208 Science Park, Milton Road, \\ Cambridge CB4 0GZ, United Kingdom \\ ${ }^{2}$ Cavendish Laboratory, Cambridge University, J. J. Thomson Avenue, Cambridge CB3 OHE, United Kingdom
}

(Received 30 January 2019; revised manuscript received 14 May 2019; published 6 June 2019)

\begin{abstract}
We exploit the nonlinearity arising from the spin-photon interaction in an InAs quantum dot to demonstrate phase shifts of scattered light pulses at the single-photon level. Photon phase shifts of close to $90^{\circ}$ are achieved using a charged quantum dot in a micropillar cavity. We also demonstrate a photon phase switch by using a spin-pumping mechanism through Raman transitions in an in-plane magnetic field. The experimental findings are supported by a theoretical model that explores the dynamics of the system. Our results demonstrate the potential of quantum-dot-induced nonlinearities for quantum information processing.
\end{abstract}

DOI: 10.1103/PhysRevApplied.11.061001

\section{INTRODUCTION}

Semiconductor quantum dots (QDs) are considered a promising candidate for quantum information processing. As excellent sources of single photons, they demonstrate unparalleled brightness [1], near perfect indistinguishability [2,3], and excellent efficiency [4]. They can be embedded in a variety of nanophotonic structures for enhanced light-matter interaction [5-7]. The nonlinear effects arising from the interaction between a photon and a single charge spin confined in a QD can be used to achieve a range of quantum operations required for quantum information processing. To that aim, spin-photon entanglement has recently been demonstrated [8], while other applications such as logic operations [9-12] have been proposed.

There has been a significant effort to exploit the nonlinearities arising from spin-photon interactions to realize a quantum switch. Proposals have been made to make a spin-photon switch using an emitter in a cavity $[13,14]$. Typically, this relies on the rotation of the polarization of a photon coherently scattered by the single spin, inducing photon phase shifts $\phi$ up to $180^{\circ}$. This so-called giant Faraday or Kerr rotation began to attract attention in the 1980s, when theoretical proposals suggested utilizing the phenomenon to achieve optical quantum nondemolition

\footnotetext{
*1w585@cam.ac.uk

${ }^{\dagger}$ Current affiliation: Institute for Compound Semiconductors, Cardiff University, Queen's Buildings, 5 The Parade, Roath, Cardiff CF24 3AA, United Kingdom

$¥$ Current affiliation: Department of Electronic \& Electrical Engineering, University of Sheffield, Mappin Street, Sheffield S1 3JD, United Kingdom
}

measurements [15-17]. Measurements of Kerr and Faraday rotations using QDs were not reported until much later, with rotation angles in the few $10^{-3}$ degree range [18-20] reported on charged QD systems under a Faraday geometry magnetic field. Significant improvement on the rotation angles have been reported recently, with rotations of $6^{\circ}$ recorded for a QD strongly coupled to a micropillar cavity at $T=20 \mathrm{~K}$ [21]. More recently, rotations of more than $90^{\circ}$ have been reported for a QD in a "bad" cavity in a Faraday geometry magnetic field [22]. A quantum phase switch using a QD was demonstrated for the first time in 2016, using a QD strongly coupled to a photonic-crystal defect cavity in a Voigt geometry magnetic field [23]. While two-dimensional (2D) photonic-crystal cavities can offer high $Q$ factors and integration with on-chip quantum photonic circuits, they have low photon extraction efficiencies compared to micropillar structures [24]. Furthermore, achieving strong light-matter coupling is demanding, with limited reproducibility considering current nanofabrication processes.

Here, we demonstrate a photon phase switch using a charged QD weakly coupled to the confined mode of a micropillar cavity. Using a Voigt geometry magnetic field, we demonstrate phase shifts of $80 \pm 2^{\circ}$ of coherently scattered laser pulses that, on average, contain approximately one photon. After preparing the spin in an eigenstate [25], we use a second pulse to switch the QD-induced phase shift of the initial pulse on and off. Finally, we develop a theoretical model that provides further insight into performance-limiting factors. We show that polarization control is possible using a transition in a single quantum dot [26]. 


\section{CONCEPT AND EXPERIMENTAL SETUP}

The core of our phase-switching system is a singly charged InAs quantum-dot held in a $B=8 \mathrm{~T}$ magnetic field (Voigt geometry) [27]. Its energy levels form a double-lambda system [Fig. 1(c)]. $|G\rangle$ and $|\bar{G}\rangle(|T\rangle$ and $|\bar{T}\rangle)$ represent orthogonal ground (trion) states. The QD is in a $2.75 \mu \mathrm{m}$ diameter micropillar cavity, with the top (bottom) mirror consisting of 17 (25) mirror pairs. This increases the light-collection efficiency and enhances emission from the long-wavelength vertical transition of interest through weak coupling with the cavity mode, with Purcell factor $F_{P} \approx 2$ and cooperativity $C=\frac{1}{2} F_{P} \approx 1$ [28]. The cavity-mode quality factor is $Q \approx 5000$. The vertical and diagonal transitions couple to orthogonal linear polarizations of light, represented by $V$ and $H$, respectively. The long-wavelength vertical transition is excited using resonant right-handed circularly polarized laser pulses. The quantum state of the photon can be written as $\left|\phi_{i}\right\rangle=|H\rangle+i|V\rangle$ [29]. On reflection, the state becomes $\left|\phi_{f}\right\rangle_{\uparrow(\downarrow)}=|H\rangle+i r_{\uparrow(\downarrow)}|V\rangle$, where $r_{\uparrow}\left(r_{\downarrow}\right)$ is the reflection coefficient for the QD in the spin-up (spin-down) state. The observed phase change is dependent on the interference contrast $\alpha=\kappa_{\mathrm{ex}} / \kappa$, where $\kappa_{\mathrm{ex}}$ and $\kappa$ are the cavity energy decay rate to the reflected mode and the total cavity energy decay rate. When $\alpha>0.5$ and $C>2 \alpha-1, r_{\uparrow}$ and $r_{\downarrow}$ have opposite signs. Hence the incident photon experiences a spin-state-conditional $180^{\circ}$ phase shift [30]. Fitting the reflectivity spectrum of our cavity allows us to extract a value of $\alpha=0.93$ [23]. We define the polarization contrast as $P=\left(I_{R}-I_{L}\right) /\left(I_{R}+I_{L}\right)$, where $I_{R}$ and $I_{L}$ are the scattered intensities of right-circularly polarized (RCP) and left-circularly polarized (LCP) light, respectively. The QD-induced photon phase shift is determined by $\phi=\cos ^{-1}(P)$.

The experimental setup is illustrated in Fig. 1(a). The enhanced vertical $|\bar{G}\rangle \rightarrow|T\rangle$ transition is probed using a narrow-line-width laser at $934.55 \mathrm{~nm}$, referred to as the
"Target." A second narrow-line-width laser at $934.49 \mathrm{~nm}$ can be used to drive the diagonal $|\bar{G}\rangle \rightarrow|\bar{T}\rangle$ transition and is termed the "Control" [Fig. 1(c)]. Emission from these lasers is controlled via amplitude modulators to achieve coherent pulses [250 ps (7 ns) width for the "Target" ("Control")] with an $80 \mathrm{MHz}$ repetition rate. The excitation pulses are RCP. A series of polarization optics and a polarizing beam splitter in the detection path allow us to record $I_{R}$ and $I_{L}$ simultaneously to measure the individual contribution of each polarization to the total collected light. As the sample is nominally undoped, we use a weak nonresonant pulsed laser to inject a charge with random spin into the QD [26]. Both positive and negative trions are created in this way, although only one is resonant with the cavity and observed here (for further details, see the Supplemental Material [31]). The photoluminescence spectrum under nonresonant excitation at $B=8 \mathrm{~T}$ is shown in Fig. 1(d). We observe four distinct peaks that are assigned to the vertical and diagonal transitions, with the longest-wavelength transition enhanced due to weak coupling with the cavity mode. The inferred $g$ factor is $|0.86|(|0.18|)$ for the ground states (excited states). These values are comparable with previous reports detailing both electrons and holes in the ground state [32-34]. It is not possible to assign a specific carrier to the $g$ factors from Fig. 1(d) and our analysis. Since the injected charge type does not impact the mechanisms used for this experiment, we do not specify whether an electron or hole is captured. The temperature is used to tune the long-wavelength vertical transition in resonance with the cavity mode. The sample is therefore held at $T=18 \mathrm{~K}$.

\section{QUANTUM-DOT-INDUCED PHOTON PHASE SHIFT}

After injecting a charge into the QD with the weak nonresonant laser pulse, we probe the change in photon phase induced by the cavity-coupled QD transition [Fig. 2(a)].

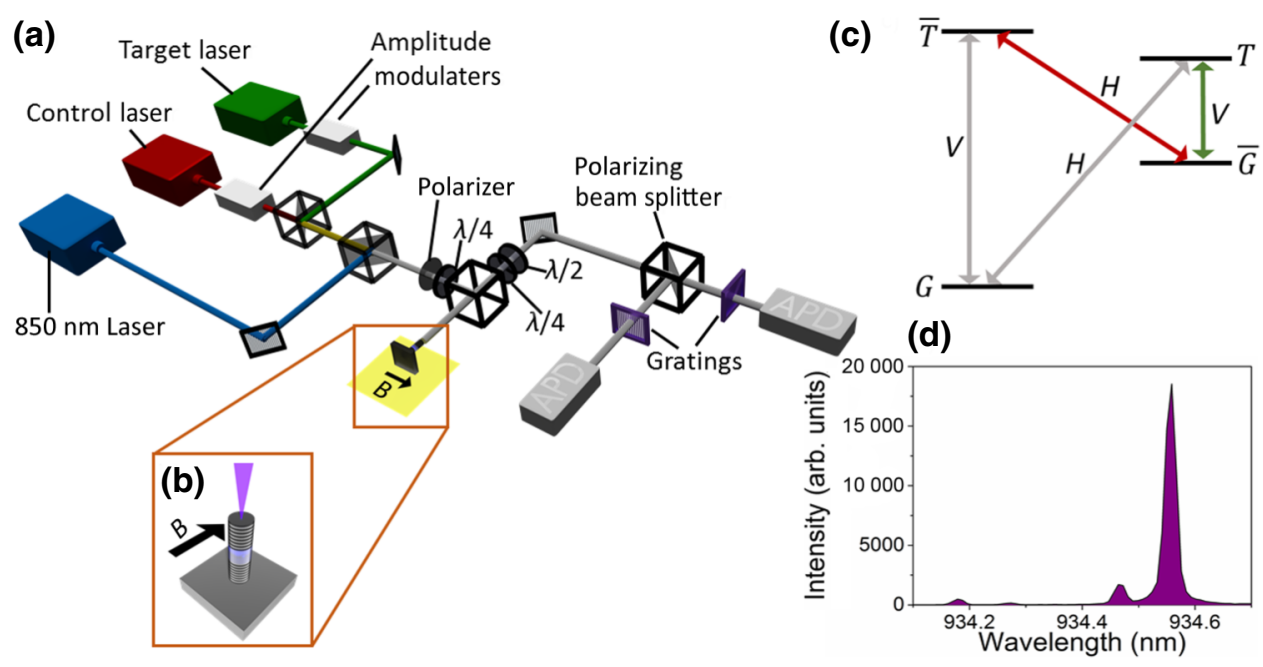

FIG. 1. (a) The experimental setup. (b) A micropillar cavity in a Voigt geometry magnetic field. (c) The energy-level diagram, showing the cavity-enhanced "Target" transition (green arrow) and "Control" transition (red arrow). (d) The photoluminescence spectrum of the QD under nonresonant excitation. The long-wavelength transition is enhanced by the cavity mode. 


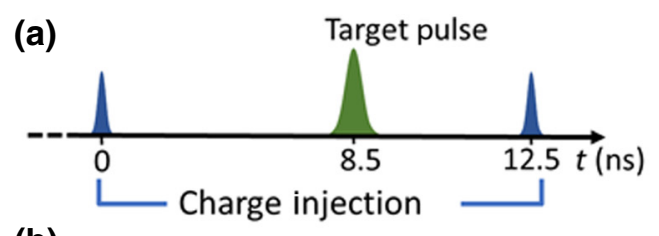

(b)

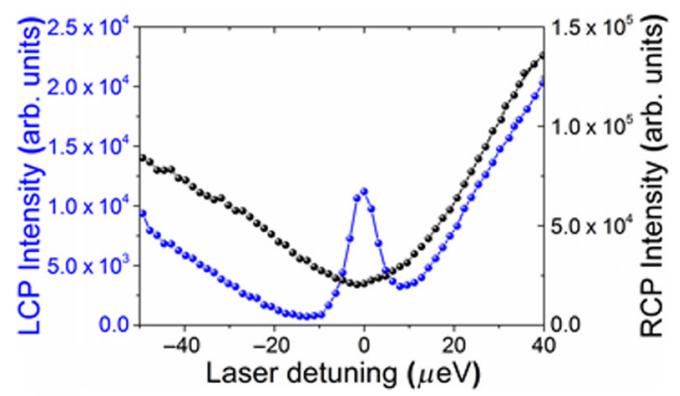

(c)

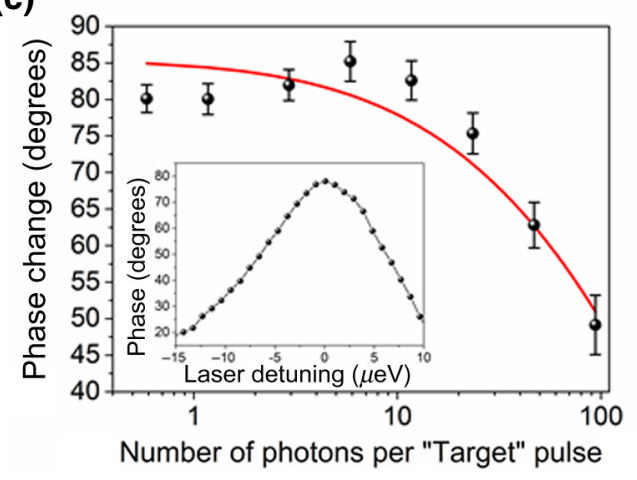

FIG. 2. (a) The pulse sequence used to phase shift the reflected photons. (b) The reflectivity spectrum of the cavity for LCP (blue) and RCP (black) light, with an average of 12 photons per "Target" pulse. (c) The recorded phase shift as a function of the average number of photons per "Target" pulse (black data points) and the calculated phase shift (red line). The inset shows the phase change as a function of the laser detuning for an average of 12 photons per "Target" pulse.

The system is probed using the "Target" pulse and we measure the RCP and LCP components of the reflected signal [Fig. 2(b)]. The RCP reflected light shows the cavity response and does not contain any indication that the incident photon interacted with the QD spin. In the LCP channel, we observe a modification of the cavity reflectivity when the laser is on resonance with the QD transition. The observed peak is due to resonance fluorescence and is a manifestation of the QD phase shift. The marginal split (approximately $6 \mu \mathrm{eV}$ ) observed between the cavity centers recorded in the two polarizations is attributed to the small ellipticity of the micropillar acquired during the fabrication process. We note that the observed peak measures $10 \mu \mathrm{eV}$ in width and is broad compared to the results reported in other publications. Several factors may contribute to this, including the width of the "Target" pulse (approximately $4 \mu \mathrm{eV}$ ), the Purcell effect, which reduces the lifetime of the QD [11], and spectral jittering.
The QD-induced phase shift as a function of the laser detuning is shown in the inset of Fig. 2(c). It is extracted using the reflected RCP and LCP signals in Fig. 2(b). The data are fitted with a Gaussian function and the peak of this curve is used to obtain the maximum achieved phase shift. The resulting maximal QD-induced phase shift of the "Target" pulses as a function of the average photon number per pulse is shown in Fig. 2(c). At the limit of a single photon on average per "Target" pulse, we observe phase shifts of $80 \pm 2^{\circ}$. Given that the random nature of charge initialization in our system results in occupation of both ground states with equal probability, the observed photon phase shifts are close to the expected value of $90^{\circ}$. Intrinsic effects, such as spectral jitter, may limit the induced phase shifts [24]. Furthermore, the cavity is birefringent, as can be seen in the different energies of the modes, which may introduce a degree of ellipticity in the incident RCP "Target" pulse [35]. As the average photon number per pulse increases, we observe a decrease of the recorded phase shifts. We attribute this to the collected RCP intensity increasing more rapidly than the collected LCP intensity as the number of photons per pulse increases. We model this by considering the decreasing contribution of the coherently scattered photons to the total scattered intensity as a function of the incident power following a previously developed model [36]. We use the measured value of $T_{1}=0.5 \mathrm{~ns}$ for the transition lifetime and the coherence time $T_{2}=1 \mathrm{~ns}$ as a fitting parameter. Such a high value for the coherence time is reasonable given the coherent nature of the photon-scattering event [36]. The result of this model is shown in Fig. 2(c) (red line) and is in good agreement with our experimental results.

\section{QUANTUM-DOT-INDUCED PHOTON PHASE SWITCH}

To demonstrate a QD-induced phase switch, we introduce a "Control" pulse. The pulse sequence works as follows [Fig. 3(a)]. The nonresonant weak laser pulse injects a charge into the QD. The "Control" pulse drives the diagonal $|\bar{G}\rangle \rightarrow|\bar{T}\rangle$ transition, pumping the charge out of the ground state of the $|\bar{G}\rangle \rightarrow|T\rangle$ transition. This pulse duration is relatively long $(7 \mathrm{~ns})$ at a relatively high power (average 940 photons per pulse) to achieve efficient spin pumping. The "Target" pulse is used to probe the $|\bar{G}\rangle \rightarrow$ $|T\rangle$ transition and the phase change is measured. Without a charge in the $|\bar{G}\rangle$ ground state, the "Target" photon will not interact with the QD and will be reflected without changing phase. The "Control" pulse can be switched on and off, enabling control over the observed phase shift. As the two driven transitions are very close (approximately $130 \mu \mathrm{eV}$ ), extremely narrow-band spectral filtering is introduced. This suppresses the contribution of the "Control" pulse to the recorded signal. 

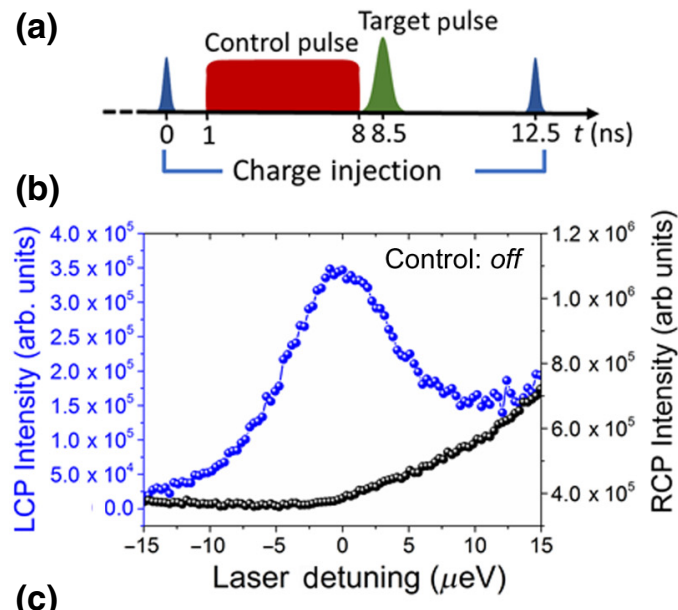

(c)

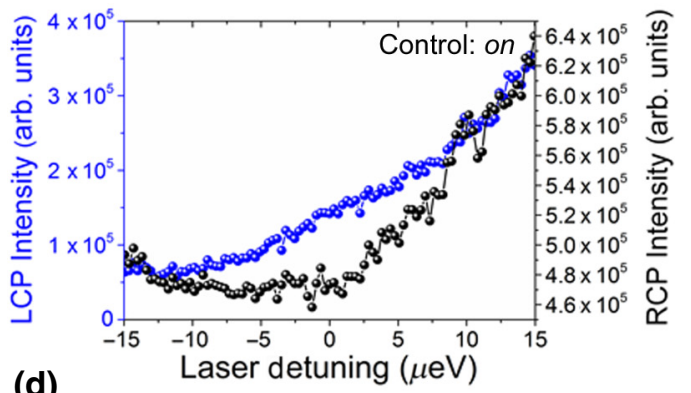

(d)

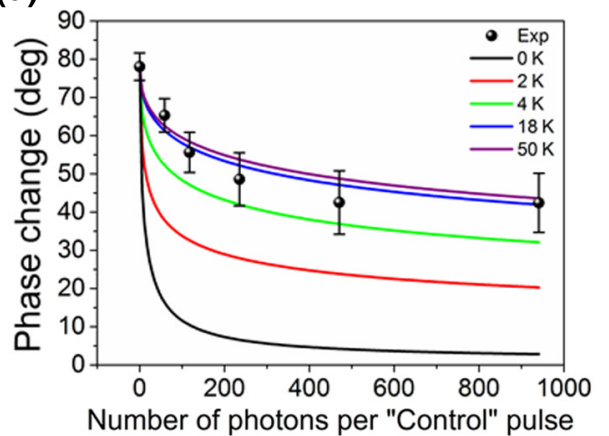

FIG. 3. (a) The pulse sequence used to generate and control the phase shift of the reflected photons. (b) The reflectivity spectrum of the system for RCP (black) and LCP (blue) light when the "Control" pulse is off and (c) when the "Control" pulse is on. The differences in the changes in the LCP and RCP signals come about as a result of a marginal offset between the respective cavity-mode centers. (d) The measured phase change as a function of the number of photons per "Control" pulse (black data points) and the calculated phase change for several different temperatures (solid lines).

The spectra for both components of the reflected signal with the "Control" pulse is off (on) are shown in Fig. 3(b) [Fig. 3(c)]. In the absence of the control pulse, the LCP reflected signal is enhanced when the driving laser is on resonance with the "Target" transition, similar to Fig. 2(b). Introduction of the "Control" pulse removes this peak almost entirely, effectively acting as a switch and turning the phase rotation off. We find that the presence of the
"Control" pulse causes a $46 \%$ reduction in the measured phase change, from $78 \pm 4^{\circ}$ to $42 \pm 8^{\circ}$.

Finally, we vary the average power of the "Control" laser pulse to investigate its impact on our switching mechanism. In Fig. 3(d), we plot the phase shift for range of "Control" pulses, with average photon numbers per pulse between 0 and 940. As expected, the phase shift reduces as the number of photons per pulse increases. The induced phase shift reaches a steady value of $42 \pm 8^{\circ}$ once there are 470 or more photons per pulse. This is a far smaller reduction than expected, considering that a successful spin-pumping mechanism should eliminate the population in the $|\bar{G}\rangle$ state [19].

To better understand the limiting mechanisms of our photon phase switch, we develop a simple theoretical model based on a system of rate equations for the relevant state populations. We calculate the polarization contrast (and hence the phase shift) using the ratio $\Xi=\left(N_{\bar{G}}^{i}-N_{\bar{G}}^{f}\right) /\left(N_{\bar{G}}^{i}+N_{\bar{G}}^{f}\right)$, where $N_{\bar{G}}^{i}$ and $N_{\bar{G}}^{f}$ are the populations of the $|\bar{G}\rangle$ state before and after the "Control" pulse, respectively (for further details of the theoretical model, see the Supplemental Material [31] and [37-41]). The calculated phase shifts as a function of the average photon number per "Control" pulse for different temperatures are shown in Fig. 3(d). This suggests that temperature-dependent mechanisms, such as spin-flip, and phonon-assisted transitions are the main factors limiting the achieved switching ratio. In particular, we experimentally achieve a phase-switching ratio of $46 \%$ for an average of 940 photons per "Control" pulse [corresponding to the $36^{\circ}$ phase shift in Fig. 3(d)]. Without a spin-flip mechanism $(T=0 \mathrm{~K})$, the same ratio is predicted to be $90 \%$ [the $76^{\circ}$ phase shift in Fig. 3(d)]. This is limited by the ellipticity in polarization of the "Target" pulse and the imperfect degeneracy of the cavity modes. For $T=18 \mathrm{~K}$, we obtain good agreement between the experimental and theoretical values [blue line in Fig. 3(c)]. We anticipate that experiments performed at lower temperatures or with a higher Zeeman splitting would reduce the spin-flip rate and therefore improve the switching contrast. We also anticipate that these changes to our system would allow us to significantly reduce the number of photons per "Control" pulse.

\section{CONCLUSIONS}

We demonstrate photon phase shifts up to $80 \pm 2^{\circ}$ at the single-photon level by exploiting nonlinear photonspin interactions in a charged QD in a micropillar cavity. We use the Raman transitions allowed due to an external magnetic field to demonstrate controllable switching of the obtained phase shifts. Limitations of the switching mechanism in our system are highlighted by a simple theoretical model based on the rate equations of the relevant states. Our findings highlight the importance of the QD-micropillar-cavity system as a nonlinear medium 
for developing photonic quantum-logic operations toward quantum information processing. The experimental data used to produce the figures in this paper is publicly available [42]

\section{ACKNOWLEDGMENTS}

We acknowledge funding from the Engineering and Physical Sciences Research Council (EPSRC) for the molecular-beam-epitaxy (MBE) system used to grow the micropillar cavity. L.W. gratefully acknowledges funding from the EPSRC and financial support from Toshiba Research Europe Ltd. B.V. gratefully acknowledges funding from the European Union's Horizon 2020 Research and Innovation program under the Marie SkłodowskaCurie Grant Agreement No. 642688 (SAWtrain).

[1] Sebastian Maier, Peter Gold, Alfred Forchel, Niels Gregersen, Jesper Mørk, Sven Höfling, Christian Schneider, and Martin Kamp, Bright single photon source based on self-aligned quantum dot-cavity systems, Opt. Express 22, 8136 (2014).

[2] N. Somaschi, V. Giesz, L. de Santis, J. C. Loredo, M. P. Almeida, G. Hornecker, S. L. Portalupi, T. Grange, C. Antón, J. Demory, C. Gómez, I. Sagnes, N. D. LanzillottiKimura, A. Lemaítre, A. Auffeves, A. G. White, L. Lanco, and P. Senellart, Near-optimal single-photon sources in the solid state, Nat. Photonics 10, 340 (2016).

[3] Xing Ding, Yu He, Z.-C. Duan, Niels Gregersen, M.-C. Chen, S. Unsleber, S. Maier, Christian Schneider, Martin Kamp, Sven Höfling, Chao-Yang Lu, and Jian-Wei Pan, On-Demand Single Photons with High Extraction Efficiency and Near-Unity Indistinguishability from a Resonantly Driven Quantum Dot in a Micropillar, Phys. Rev. Lett. 116, 020401 (2016).

[4] Julien Claudon, Joël Bleuse, Nitin Singh Malik, Maela Bazin, Christophe Sauvan, Philippe Lalanne, and JeanMichel Gérard, A highly efficient single-photon source based on a quantum dot in a photonic nanowire, Nat. Photonics 4, 174 (2010).

[5] J. M. Gérard, D. Barrier, J. Y. Marzin, R. Kuszelewicz, L. Manin, E. Costard, V. Thierry-Mieg, and T. Rivera, Quantum boxes as active probes for photonic microstructures: The pillar microcavity case, Appl. Phys. Lett. 69, 449 (1996).

[6] A. Kiraz, P. Michler, C. Becher, B. Gayral, A. Imamoğlu, Lidong Zhang, E. Hu, W. V. Schoenfeld, and P. M. Petroff, Cavity-quantum electrodynamics using a single InAs quantum dot in a microdisk structure, Appl. Phys. Lett. 78, 3932 (2001).

[7] Jelena Vučković and Yoshihisa Yamamoto, Photonic crystal microcavities for cavity quantum electrodynamics with a single quantum dot, Appl. Phys. Lett. 82, 2374 (2003).

[8] E. Togan, Y. Chu, A. S. Trifonov, L. Jiang, J. Maze, L. Childress, M. V. G. Dutt, A. S. Sørensen, P. R. Hemmer, A. S. Zibrov, and M. D. Lukin, Quantum entanglement between an optical photon and a solid-state spin qubit, Nature 466, 730 (2010).
[9] Cristian Bonato, Florian Haupt, Sumant S. R. Oemrawsingh, Jan Gudat, Dapeng Ding, Martin P. van Exter, and Dirk Bouwmeester, CNOT and Bell-State Analysis in the Weak-Coupling Cavity QED Regime, Phys. Rev. Lett. 104, 160503 (2010).

[10] T. C. Ralph, I. Söllner, S. Mahmoodian, A. G. White, and P. Lodahl, Photon Sorting, Efficient Bell Measurements, and a Deterministic Controlled- $z$ Gate Using a Passive Two-Level Nonlinearity, Phys. Rev. Lett. 114, 173603 (2015).

[11] A. J. Bennett, J. P. Lee, D. J. P. Ellis, I. Farrer, D. A. Ritchie, and A. J. Shields, A semiconductor photon-sorter, Nat. Nanotechnol. 11, 857 (2016).

[12] C. Y. Hu, Photonic transistor and router using a single quantum-dot-confined spin in a single-sided optical microcavity, Sci. Rep. 7, 45582 (2017).

[13] L.-M. Duan and H. J. Kimble, Scalable Photonic Quantum Computation Through Cavity-Assisted Interactions, Phys. Rev. Lett. 92, 127902 (2004).

[14] Kae Nemoto and W. J. Munro, Nearly Deterministic Linear Optical Controlled-NOT Gate, Phys. Rev. Lett. 93, 250502 (2004).

[15] P. Grangier, J. F. Roch, and S. Reynaud, Quantum correlations and non-demolition measurements using two-photon non-linearities in optical cavities, Opt. Commun. 72, 387 (1989).

[16] Philippe Grangier, Juan Ariel Levenson, and Jean-Philippe Poizat, Quantum non-demolition measurements in optics, Nature 396, 537 (1998).

[17] Y. Takahashi, K. Honda, N. Tanaka, K. Toyoda, K. Ishikawa, and T. Yabuzaki, Quantum nondemolition measurement of spin via the paramagnetic Faraday rotation, Phys. Rev. A 60, 4974 (1999).

[18] J. Berezovsky, M. H. Mikkelsen, O. Gywat, N. G. Stoltz, L. A. Coldren, and D. D. Awschalom, Nondestructive optical measurements of a single electron spin in a quantum dot, Science 314, 1916 (2006).

[19] Mete Atatüre, Jan Dreiser, Antonio Badolato, and Atac Imamoglu, Observation of Faraday rotation from a single confined spin, Nat. Phys. 3, 101 (2007).

[20] M. H. Mikkelsen, J. Berezovsky, N. G. Stoltz, L. A. Coldren, and D. D. Awschalom, Optically detected coherent spin dynamics of a single electron in a quantum dot, Nat. Phys. 3, 770 (2007).

[21] Christophe Arnold, Justin Demory, Vivien Loo, Aristide Lemaître, Isabelle Sagnes, Paul Voisin, Pascale Senellart, and Loïc Lanco, Macroscopic rotation of photon polarization induced by a single spin, Nat. Commun. 6, 6236 (2015).

[22] P. Androvitsaneas, A. B. Young, C. Schneider, S. Höfling, M. Kamp, E. Harbord, J. G. Rarity, and R. Oulton, in 2015 European Conference on Lasers and ElectroOptics-European Quantum Electronics Conference (Optical Society of America, Munich, 2015), p. EA 84 .

[23] Shuo Sun, Hyochul Kim, GlennS. Solomon, and Edo Waks, A quantum phase switch between a single solid-state spin and a photon, Nat. Nanotechnol. 11, 539 (2016).

[24] Shunfa Liu, Yuming Wei, Rongling Su, Rongbin Su, Ben Ma, Zesheng Chen, Haiqiao Ni, Zhichuan Niu, Ying Yu, Yujia Wei, Xuehua Wang, and Siyuan Yu, A deterministic 
quantum dot micropillar single photon source with $>$ $65 \%$ extraction efficiency based on fluorescence imaging method, Sci. Rep. 7, 13986 (2017).

[25] Mete Atatüre, Jan Dreiser, Antonio Badolato, Alexander Högele, Khaled Karrai, and Atac Imamoglu, Quantum-dot spin-state preparation with near-unity fidelity, Science 312, 551 (2006).

[26] J. P. Lee, L. M. Wells, B. Villa, S. Kalliakos, R. M. Stevenson, D. J. P. Ellis, I. Farrer, D. A. Ritchie, A. J. Bennett, and A. J. Shields, Controllable Photonic TimeBin Qubits from a Quantum Dot, Phys. Rev. X 8, 021078 (2018).

[27] M. A. Pooley, D. J. P. Ellis, R. B. Patel, A. J. Bennett, K. H. A. Chan, I. Farrer, D. A. Ritchie, and A. J. Shields, Controlled-NOT gate operating with single photons, Appl. Phys. Lett. 100, 211103 (2012).

[28] Lorenzo de Santis, Carlos Antón, Bogdan Reznychenko, Niccolo Somaschi, Guillaume Coppola, Jean Senellart, Carmen Gómez, Aristide Lemaître, Isabelle Sagnes, AndrewG. White, and Pascale Senellart, A solidstate single-photon filter, Nat. Nanotechnol. 12, 663 (2017).

[29] Rodney Loudon, The Quantum Theory of Light (Oxford University Press, New York, 2000).

[30] Peter Michler, Quantum Dots for Quantum Information Technologies (Springer, Cham, 2017), Vol. 237.

[31] See Supplemental Material at http://link.aps.org/supple mental/10.1103/PhysRevApplied.11.061001 for details of the equations, parameters, and methods used in the theoretical model, including Refs. [37-41].

[32] Anthony J. Bennett, Matthew A. Pooley, Yameng Cao, Niklas Sköld, Ian Farrer, David A. Ritchie, and Andrew J. Shields, Voltage tunability of single-spin states in a quantum dot, Nat. Commun. 4, 1522 (2013).

[33] C. Emary, Xiaodong Xu, D. G. Steel, S. Saikin, and L. J. Sham, Fast Initialization of the Spin State of an Electron in a Quantum Dot in the Voigt Configuration, Phys. Rev. Lett. 98, 047401 (2007).

[34] J. van Bree, A. Yu. Silov, M. L. van Maasakkers, C. E. Pryor, M. E. Flatté, and P. M. Koenraad, Anisotropy of electron and hole $g$ tensors of quantum dots: An intuitive picture based on spin-correlated orbital currents, Phys. Rev. B 93, 035311 (2016).

[35] Dapeng Ding, Martin Hayhurst Appel, Alisa Javadi, Xiaoyan Zhou, Matthias Christian Löbl, Immo Söllner, Rüdiger Schott, Camille Papon, Tommaso Pregnolato, and Leonardo Midolo et al., Coherent optical control of a quantum-dot spin-qubit in a waveguide-based spin-photon interface, arXiv:1810.06103 (2018).

[36] Anthony J. Bennett, James P. Lee, David J. P. Ellis, Thomas Meany, Eoin Murray, Frederik F. Floether, Jonathan P. Griffths, Ian Farrer, David A. Ritchie, and Andrew J. Shields, Cavity-enhanced coherent light scattering from a quantum dot, Sci. Adv. 2, e1501256 (2016).

[37] Petros Androvitsaneas, Andrew Young, Joseph Lennon, Christian Schneider, Sebastian Maier, Janna Hinchliff, George Atkinson, Edmund Harbord, Martin Kamp, and Sven Hoefling et al., in CLEO: QELS_Fundamental Science (Optical Society of America, San Jose, 2017), p. FTu4E-4.

[38] Carlos Antón, Paul Hilaire, Christian A. Kessler, Justin Demory, Carmen Gómez, Aristide Lemaître, Isabelle Sagnes, Norberto Daniel Lanzillotti-Kimura, Olivier Krebs, Niccolo Somaschi, Senellart Pascale, Lanco Loïc, Tomography of the optical polarization rotation induced by a single quantum dot in a cavity, Optica 4, 1326 (2017).

[39] Ulrich Hohenester, Arne Laucht, Michael Kaniber, Norman Hauke, Andre Neumann, Abbas Mohtashami, Marek Seliger, Max Bichler, and Jonathan J. Finley, Phononassisted transitions from quantum dot excitons to cavity photons, Phys. Rev. B 80, 201311(R) (2009).

[40] Brian D. Gerardot, Daniel Brunner, and Paul A. Dalgarno, Optical pumping of a single hole spin in a quantum dot, Nature 451, 441 (2008).

[41] J. I. Climente, C. Segarra, and J. Planelles, Spin-orbitinduced hole spin relaxation in InAs and GaAs quantum dots, New J. Phys. 15, 093009 (2013).

[42] L. M. Wells, S. Kalliakos, B. Villa, D. J. P. Ellis, R. M. Stevenson, A. J. Bennett, I. Farrer, D. A. Ritchie, and A. J. Shields, Data for: "Photon phase shift at the few-photon level and optical switching by a quantum dot in a microcavity," https://doi.org/10.17863/CAM.40128 (2019). 